\title{
Editorial
}

\section{Nanostructured Magnetic Materials}

\author{
Weichang Hao, ${ }^{1}$ Ji Shi, $^{2}$ Gang Xiang, ${ }^{3}$ and Yi Du ${ }^{4}$ \\ ${ }^{1}$ Centre of Materials Physics and Chemistry, Beihang University, Beijing 100191, China \\ ${ }^{2}$ Department of Metallurgy and Ceramics Science, Tokyo Institute of Technology, Tokyo 152-8552, Japan \\ ${ }^{3}$ Department of Physics, Sichuan University, Chengdu 610064, China \\ ${ }^{4}$ Institute for Superconducting \& Electronic Materials, University of Wollongong, Wollongong, NSW 2522, Australia
}

Correspondence should be addressed to Weichang Hao; whao@buaa.edu.cn

Received 20 January 2013; Accepted 20 January 2013

Copyright (C) 2013 Weichang Hao et al. This is an open access article distributed under the Creative Commons Attribution License, which permits unrestricted use, distribution, and reproduction in any medium, provided the original work is properly cited.

The saga of nanostructured magnetic materials (NMMs) has prevailed since the discovery of the first giant magnetoresistance (GMR) effect in metals in 1988. NMMs represent a unique system that incorporates the interplay between the properties associated with spin degrees of freedom and the nanoscaled structures, which provide a very strong platform for exploring both basic science and technical applications in the fields of solid-state physics, chemistry, materials science, and engineering. In fact, an active research field called "spintronics", which has a big overlap with NMMs, has emerged and prevailed very recently. Through manipulation of spin of electrons in solids, a wide variety of NMMs and devices have been playing a prominent role in information processing and transport in our modern life. A rich variety of materials, such as transition metals, manganite, wide bandgap semiconductors, and nanocomposites, have already been developed for generating well-controlled nanostructures with new functionalities. Many scientists believe that the 21st century will be a "Century of Spin." Nanomaterials and nanotechnologies have provided historical opportunities for research and development of novel spintronics materials and devices. NMSs manifest fascinating properties compared to the bulks because of size effect and quantum effect. Nanotechnologies have been proven to be an effective way to fabricate devices with fine nanostructures. The combination of spintronics and nanomaterials will undisputedly open new pathways in solid-state physics. The present special issue focuses on the recent development in the understanding of the synthesis, the studies on magnetic properties of nanostructures, and their potential applications based on the multiple functionalities.
In this special issue, we have a series of contributed papers that are addressing current status of the fundamental issues related to different NMMs, from either experimental or theoretical points of view. The front edge and hot spot of materials science and condensed matter physics, dilute magnetic semiconductors (DMSs), has been covered: $\mathrm{X}$. Liang et al. demonstrated a successful synthesis and characterization of DMS $\mathrm{Cu}_{2} \mathrm{FeSnS}_{4}$ nanocrystals with a novel zinc blende structure; G. Xiang's group spotlighted the wide bandgap semiconductor $\mathrm{ZnO}$ nanocrystals and discovered that the zinc vacancies themselves could induce ferromagnetism in undoped $\mathrm{ZnO}$ at room temperature. Some of the papers illustrate the research on the manganite: A. Ablat et al's paper studied the electronic structures of multiferroic $\mathrm{BiFe}_{1-x} \mathrm{Mn}_{x} \mathrm{O}_{3}$ using $\mathrm{X}$-ray absorption spectroscopy; $\mathrm{H}$. Zhao et al. demonstrated the effect of reducing time on the colossal magnetoresistance of $\mathrm{La}_{0.67} \mathrm{Sr}_{0.20} \mathrm{Cu}_{0.10 \square 0.10} \mathrm{MnO}_{3}$. In the meantime, some of the papers cover the traditional transition-metal-based nanostructures: the paper by W. Zhou investigated the optically excited spin waves in traditional transition metal systems ( $\mathrm{Co} / \mathrm{Pt}$ nanostructures); K. M. Hyie et al.s paper studied the influence of deposition time on the magnetic properties of Co-Fe nanostructures; W. Li's group spotlighted the effect of thermomagnetic treatment on the magnetic properties of $\mathrm{Co}-\mathrm{Fe}-\mathrm{B}$ systems. N. B. Ibrahim et al. reported the synthesis and magnetic property characterization of Tb-YIG. The remaining papers illustrate nanocomposite systems:; S. H. Hosseini and A. Asadnia studied the synthesis and microwave absorbing properties of polypyrrole/ $\mathrm{MnFe}_{2} \mathrm{O}_{4}$ nanocomposite; N. X. Truong et al's paper demonstrated a theoretical simulation of magnetic 
properties of $\mathrm{Nd}_{2} \mathrm{Fe}_{14} \mathrm{~B} / \alpha$-Fe, revealing the importance of the details of microstructures; W. Cai et al. studied the nanoscaled heterostructures based on transition metals and wide bandgap semiconductors (CoPt/AlN) and illustrated that the spin reorientation transitions could be caused by anisotropy variations driven by temperature change.

\title{
Acknowledgments
}

We would like to express our sincere appreciation to all the authors and coauthors and all the reviewers for their contribution to this special issue. We also thank the reviewers for their time and valuable comments.

\author{
Weichang Hao \\ Ji Shi \\ Gang Xiang \\ Yi Du
}



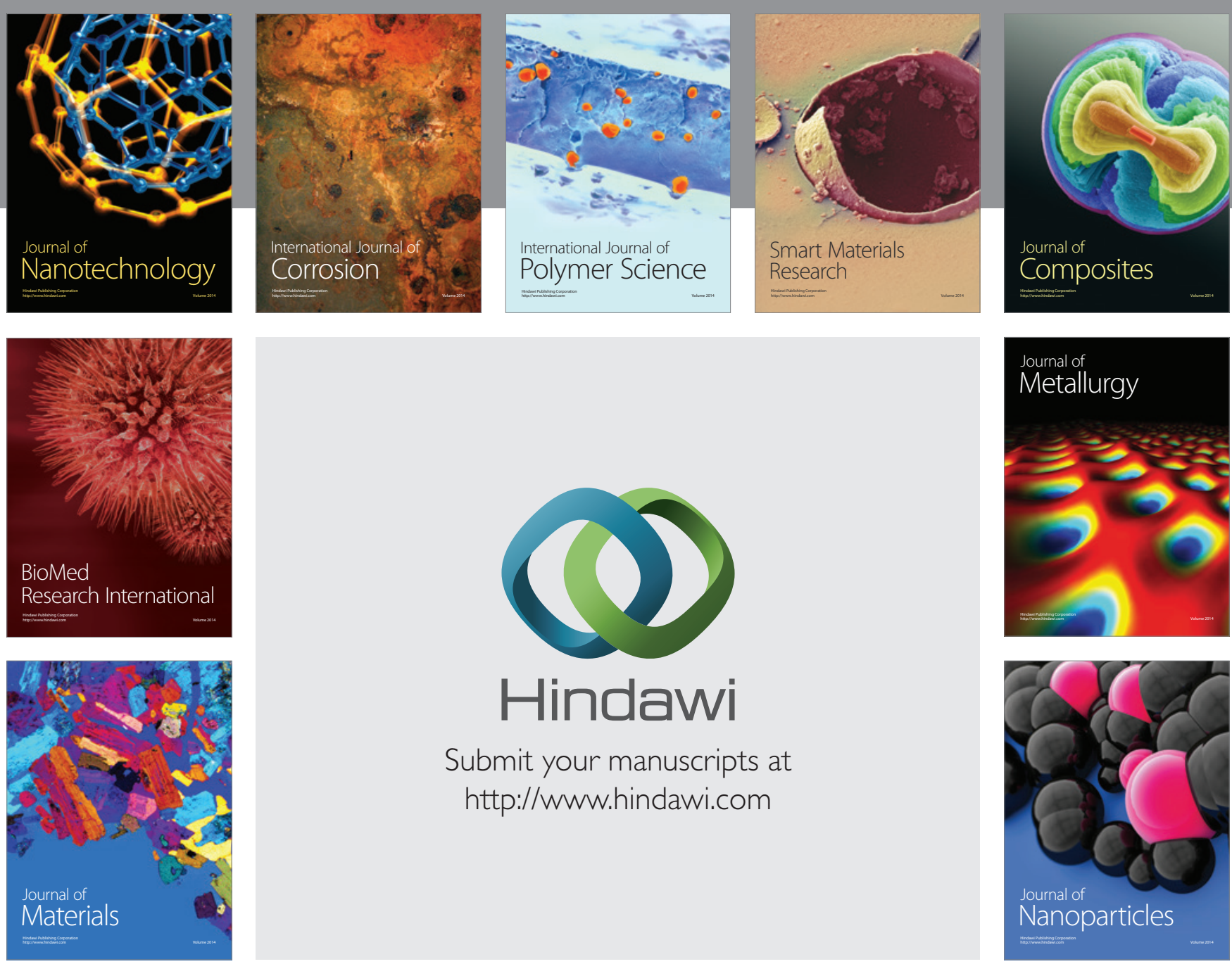

Submit your manuscripts at http://www.hindawi.com
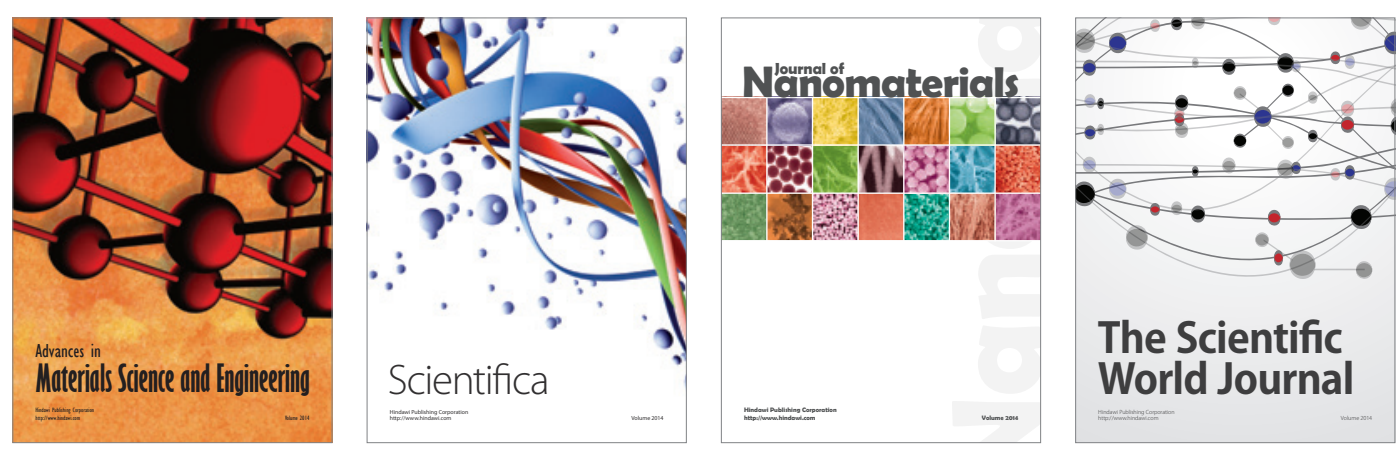

\section{The Scientific World Journal}
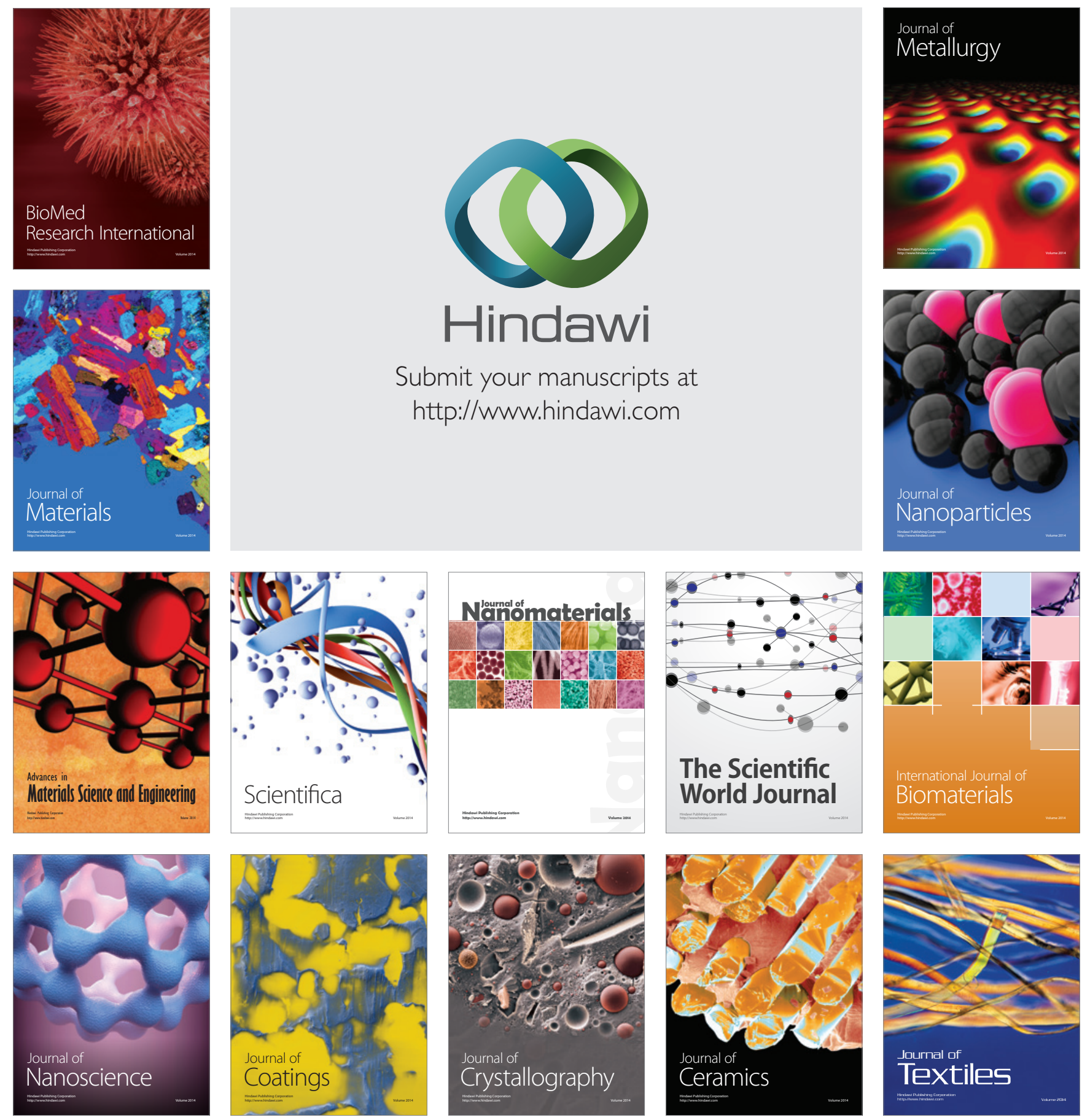